\section{Dr. Noda, et al reply}

\section{To the Editor:}

We appreciate the comments provided by Dr. Bhansing and colleagues ${ }^{1}$ in response to our article ${ }^{2}$. Recently, Bhansing, et al showed a significant 2-fold increase in the fascia thickness of the deltoid muscles on ultrasonography (US) in patients with dermatomyositis (DM) and polymyositis (PM) relative to healthy controls ${ }^{3}$. Additionally, they showed that the deltoid fascial thickness on US in patients with DM and PM was not associated with various clinical variables, including the disease duration, serum creatine kinase, the Health Assessment Questionnaire-Disability Index score, sex, antinuclear antibody, anti-Jo1, the subtype of idiopathic inflammatory myopathy, the clinical disease state, and the presence of interstitial lung disease.

In our study, the histopathological examination of fascia specimens from patients with DM and PM demonstrated that the frequency and severity of fasciitis in patients with DM was significantly higher than in patients with PM. We agree that the deltoid fascial thickness on US was increased in DM. Among the patients in our analysis, all of those who were positive for anti-Jo1 antibodies had fasciitis, irrespective of whether they had skin symptoms. The individual antiaminoacyl-tRNA synthetase antibodies, including the anti-Jo1 antibody, may be associated with fasciitis in DM and PM. In both our study and the study by Bhansing, et al, PM was diagnosed according to the Bohan and Peter criteria ${ }^{4}$. PM, as defined by the Bohan and Peter criteria, includes antisynthetase syndrome, immune-mediated necrotizing myopathy, overlap myositis, and other similar diseases. Their study population could also have included different phenotypes in the PM group. Further studies are required to confirm the association between these different phenotypes and fasciitis.

We analyzed the relationship between histopathologically proven fasciitis and the clinical variables in patients with DM and PM, and found that among the clinical variables, only myalgia (and not myositis) was associated with fasciitis. However, it may be difficult to prove whether fascial thickness on US is associated with clinical symptoms, including myalgia in patients with DM and PM. The fascia consists of 3 layers: an outer layer (collagen fibers adjacent to the subcutaneous tissue), a middle layer (massive collagen fiber bundles), and an inner layer (loose connective tissue adjacent to muscle tissue $)^{5}$. The innervation was abundantly distributed, especially in the outer and inner layers in the fascia (but not in the middle layer) ${ }^{6}$. In our previous study, fascial inflammation in DM was observed in not only the middle layer (fascia), but also the outer (epifascial tissue) and inner (subfascial tissue) layers ${ }^{7}$. The fascia on US is mainly derived from the middle layer. Moreover, the fascial thickening on US may have many causes other than fasciitis, including - but not limited to fibrosis and edema. Conversely, the increased blood flow that was detected by power Doppler ultrasonography (PDUS) is involved in the inflammation of all layers of the fascia ${ }^{8}$. Thus, the blood flow signal on PDUS, rather than fascial thickening on US, may reflect muscular symptoms. Further investigation is required to clarify this issue.

KENTARO NODA, MD, PhD; KEN YOSHIDA, MD, PhD; and DAITARO KUROSAKA, MD, PhD, Division of Rheumatology, Department of Internal Medicine, the Jikei University School of Medicine, Tokyo, Japan. Address correspondence to Dr. K. Noda, Division of Rheumatology,

Department of Internal Medicine, the Jikei University School of Medicine, 3-25-8 Nishi-shimbashi, Minato-ku, Tokyo, 105-8461, Japan.

E-mail: knoda3353@jikei.ac.jp

\section{REFERENCES}

1. Bhansing KJ, van Rosmalen MH, van Engelen BG, van Riel PL, Pillen S, Vonk MC. Ultrasound: a potential tool for detecting of fasciitis among dermatomyositis and polymyositis. J Rheumatol 2018;45:441-2.

2. Noda K, Yoshida K, Ukichi T, Furuya K, Hirai K, Kingetsu I, et al. Myalgia in patients with dermatomyositis and polymyositis is attributable to fasciitis rather than myositis: a retrospective study of 32 patients who underwent histopathological examinations. J Rheumatol 2017;44:482-7.

3. Bhansing KJ, Van Rosmalen MH, Van Engelen BG, Vonk MC, Van Riel PL, Pillen S. Increased fascial thickness of the deltoid muscle in dermatomyositis and polymyositis: an ultrasound study. Muscle Nerve 2015;52:534-9.

4. Bohan A, Peter JB. Polymyositis and dermatomyositis (second of two parts). N Engl J Med 1975;292:403-7.

5. Tesarz J, Hoheisel U, Wiedenhöfer B, Mense S. Sensory innervation of the thoracolumbar fascia in rats and humans. Neuroscience 2011;194:302-8.

6. Hoheisel U, Rosner J, Mense S. Innervation changes induced by inflammation of the rat thoracolumbar fascia. Neuroscience 2015;300:351-9.

7. Yoshida K, Kurosaka D, Joh K, Matsushima S, Takahashi E, Hirai $\mathrm{K}$, et al. Fasciitis as a common lesion of dermatomyositis, demonstrated early after disease onset by en bloc biopsy combined with magnetic resonance imaging. Arthritis Rheum 2010;62:3751-9.

8. Yoshida K, Nishioka M, Matsushima S, Joh K, Oto Y, Yoshiga M, et al. Brief report: power Doppler ultrasonography for detection of increased vascularity in the fascia: a potential early diagnostic tool in fasciitis of dermatomyositis. Arthritis Rheum 2016;68:2986-91.

J Rheumatol 2018;45:3; doi:10.3899/jrheum.171244 